\title{
Ofloxacin-induced toxic epidermal necrolysis
}

Sir,

Toxic epidermal necrolysis (TEN) is a potentially life-threatening dermatologic disorder characterized by widespread erythema, necrosis, and bullous detachment of the epidermis and mucous membranes, resulting in exfoliation and possible sepsis and/or death. It is also known as Lyell's syndrome. The drugs most commonly involved are antibiotics such as sulfonamides, beta-lactams tetracyclines, and quinolones. Anticonvulsants are such as phenytoin, phenobarbital and carbamazepine. Antiretroviral drugs, nonsteroidal anti-inflammatory drugs, and allopurinol. ${ }^{[1]}$ Mycoplasma pneumoniae, herpes simplex, lupus erythematosus, aplastic anemia after allogeneic hematopoietic stem cell transplantation have also been reported as a cause of nondrug induced TEN. ${ }^{[2]}$

A 42-year-old man presented with rapidly progressive generalized detachment of skin. Patient gave a history of abdominal pain and diarrhea for which he took tablets containing ofloxacin. After 2 days, he developed acute generalized erythematous rashes all over his body including face, trunk, upper, and lower extremities [Figure 1]. He also developed erosive lesions over oral mucosa and congestion of conjuntiva [Figure 2].

On examination, patient was febrile $\left(39^{\circ} \mathrm{C}\right)$ and hypotensive $(100 / 70 \mathrm{~mm} \mathrm{Hg})$. Generalized sheet like peeling of the skin involving $>90 \%$ of body was present. Erosion and crusting over lip was present. Nikolsky sign was positive. There was no hepatosplenomegaly or lymphadenopathy.

His total white blood cell count was $5.23 \times 10^{9} / \mathrm{L}$. Platelet count was normal. Hemoglobin was $13.5 \mathrm{~g} / \mathrm{dl}$. Liver function tests showed mild elevation in aspartate aminotransferase $(90 \mathrm{U} / \mathrm{L})$ and alanine aminotransferase $(70 \mathrm{U} / \mathrm{L})$. Serum creatinine was $1.1 \mathrm{mg} / \mathrm{dl}$.

History and clinical findings were suggestive of TEN secondary to ofloxacin intake. Patient was treated with dexamethasone intravenously and fusidic acid antibiotic cream topically. Fluid management, symptomatic and supportive management had been done. Patient showed significant improvement within 2 week. Lesions totally resolved in a few weeks leaving postinflammatory pigmentation over body. 


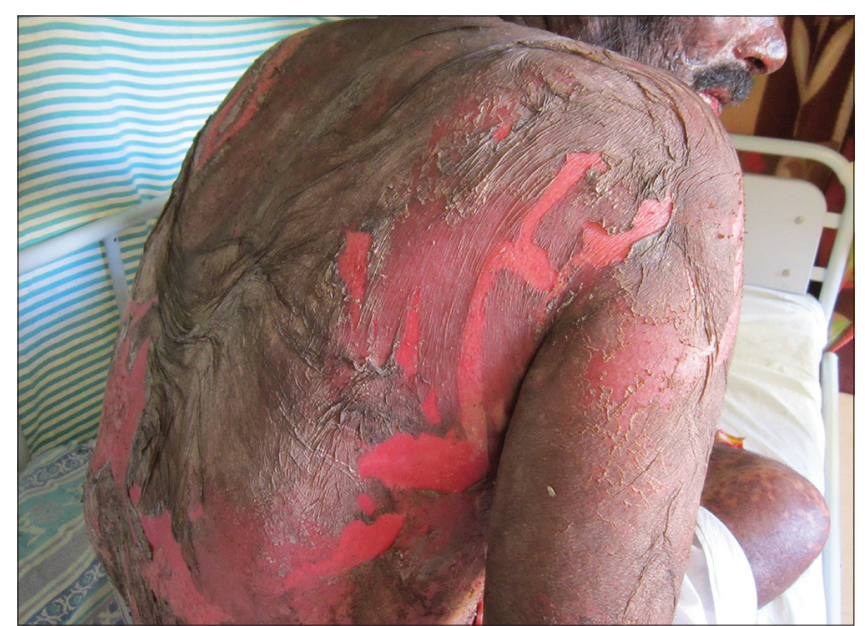

Figure I: Sheet like peeling of skin

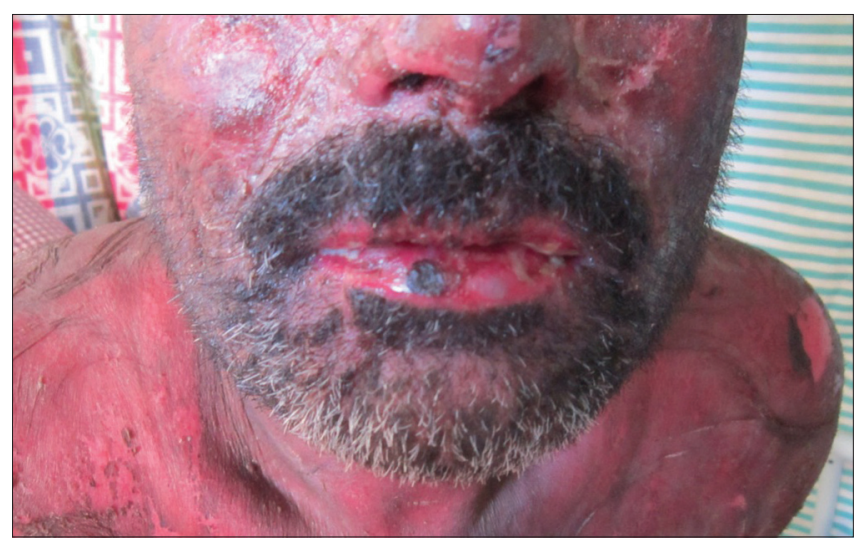

Figure 2: Crusting and erosive lesions over lip

The pathogenesis of Stevens-Johnson syndrome (SJS)/TEN is not fully understood, but is believed to be immune-mediated. The clinical, histopathological, and immunological findings in SJS/TEN support the concept, that SJS and TEN are specific drug hypersensitivity reactions in which cytotoxic $\mathrm{T}$ lymphocytes play a role in the initiation phase. ${ }^{[3]}$ The histopathology of SJS/TEN lesions show that keratinocyte apoptosis followed by necrosis is the pathogenic basis of the widespread epidermal detachment observed in SJS/TEN.. ${ }^{[2]}$ The risk of death for patients with TEN can be accurately predicted by the TEN-specific severity-of-illness score. We calculated that our patient had a final score of 2 and a mortality risk of $12.1 \%$.

The frequency of fluoroquinolone use has been increasing owing to their broad antibacterial spectra and few adverse effects. Until now, these drugs have seldom been implicated in patients with TEN, but the incidence of fluoroquinolone-induced cutaneous reactions may be on the increase. ${ }^{[4]}$ Until date, a total of 31 cases of SJS or TEN in which a fluoroquinolone was the offending agent have been reported. Of these, 26 were associated with ciprofloxacin, whereas only one was associated with ofloxacin. ${ }^{[5]}$

Here, we are reporting a patient with TEN induced by ingestion of oral ofloxacin which is not a common cause of TEN.

Gaurang Gupta
Department of dermatology, Om Premia Hospital, Palwal,
Haryana, India
Correspondence:
Dr. Gaurang Gupta,
Department of Dermatology, Om Premia Hospital, 56 km stone,
Palwal, Haryana India.
Email: gauranggups@gmail.com

\section{References}

1. Lissia M, Mulas P, Bulla A, Rubino C. Toxic epidermal necrolysis (Lyell's disease). Burns 2010;36:152-63.

2. Harr T, French LE. Toxic epidermal necrolysis and Stevens-Johnson syndrome. Orphanet J Rare Dis 2010;5:39.

3. Correia O, Delgado L, Ramos JP, Resende C, Torrinha JA. Cutaneous T-cell recruitment in toxic epidermal necrolysis. Further evidence of CD8+lymphocyte involvement. Arch Dermatol 1993;129:466-8.

4. Melde SL. Ofloxacin: A probable cause of toxic epidermal necrolysis. Ann Pharmacother 2001;35:1388-90.

5. Van Bambeke F, Tulkens PM. Safety profile of the respiratory fluoroquinolone moxifloxacin: Comparison with other fluoroquinolones and other antibacterial classes. Drug Saf 2009;32:359-78.

\begin{tabular}{|l|l|}
\hline \multicolumn{2}{|c|}{ Access this article online } \\
\hline Quick Response Code: & Website: \\
\hline & www.ijccm.org \\
\cline { 2 - 3 } & Dol: 10.4103/0972-5229.138166 \\
\hline
\end{tabular}

